\title{
Automatic adjustment of image processing pipeline
}

\author{
D.A. Kolchaev ${ }^{1}$, E.R. Muratov ${ }^{1}$, M.B. Nikiforov ${ }^{1}$ \\ ${ }^{1}$ Ryazan State Radio Engineering University, Gagarina, 59/1, 390005, Ryazan, Russia
}

\begin{abstract}
Mathematical processing of images in real-time vision systems can be conventionally divided into two stages: preprocessing (filtering, contrasting, protection from natural distortions, etc.) and final one (imposition, visualization, solution of the navigation task, etc.). Mentioned tasks can be solved by a lot of known and specially developed methods with various degrees of efficiency. The present paper suggests a mathematical criterion model and algorithm of automatic selection of the most effective method at each stage of the image processing pipeline in relation to the current situation at its input.
\end{abstract}

Keywords: image processing pipeline; real time; automatic algorithm selection

\section{Introduction}

Important stage in vision systems is preprocessing which includes contrasting of images and compensation of interferences. Since contemporary algorithms of enhancement provide different results which depend on both processed image and control parameters transmitted to these algorithms then there is a necessity of dynamical selection of the algorithm depending on the plot. Combination of all variants of algorithms and also all variants for control parameters provides a set of solutions with high dimensionality. Solution of this issue is usage of the automatic system for selection of enhancement algorithms and also selection of optimal control parameters for the series of $t$ frames obtained from the video sequence [1].

\section{Task definition}

Let a set of contrasting algorithms be $\mathrm{A}$ and a set $\mathrm{B}$ be variations of the method for interference compensation then $\mathrm{A}_{\mathrm{i}}$ is $i$ algorithm of contrasting and $\mathrm{B}_{\mathrm{j}}$ is $j$-variation of the interference compensation method. Hence, direct multiplication of a set $\mathrm{A}$ by a set $B$ provides a set $M$ which describes all possible variants of the mutual application of these algorithms. $M=A \times B .\left(A_{1}, B\right)$ is one of selection variants, $\left(A_{1}, B_{1}\right) \in M$. Initial task is the following: to find such element $\left(A_{i}, B_{j}\right)$ from the set $M$ when quality index of the resulting image is the best and noise index is located in the range corresponding to algorithm $\mathrm{B}_{\mathrm{j}}$. For current task we have $i, j=0 . .3$ because three algorithms of contrasting and three variants of noise time filtering usage have been selected experimentally.

\section{Algorithm for automatic selection of combination of methods for image enhancement and interference compensation}

Algorithm allows finding the best variant of processing by selecting a certain combination providing the best result from the point of view of some objective index from a set of interference compensation methods with various control parameters and from several algorithms of image contrasting. Processing results are estimated after every $t$ frames after accumulation of the sequence of four reference frames where the best processing algorithms correspond to each frame. Frequency of these algorithm repetitions is estimated and algorithms which repetition frequency is the highest are selected. The algorithm allows selecting the best element from the set $\mathrm{M}$ for each frame and recording this element to the stack. When the stack is filled and the most effective processing algorithm is selected, it is applied to the current image. After following $\mathrm{n}$ frames the element which was first recorded for the stack is removed and the best element from the set M occupies the stack top. Procedure of selection of application of the algorithm is repeated. Stack is organized according to the principle FILO (First-In-Last-Out).

Main blocks of the algorithm are a block of quality assessment and block of noise evaluation.

Integral performance index (IPI) is calculated as an amount of average brightness, brightness root-mean-square deviation, normalized contrast index, number of informative levels and entropy. Number of tonal gradations characterizes a number of various informative levels being present on the image and it is determined by the image histogram [2]:

$$
G\left(Z_{i}\right)=\left\{\begin{array}{l}
0, \text { if } Z_{i}=0 \\
1, \text { if } Z_{i}>0
\end{array}\right.
$$

where $Z_{i}$ - a number of point which brightness is equal to $i$.

One of the most significant characteristics of the image is average brightness $\bar{L}$ [3] calculated according to the following formula:

$$
\bar{L}=\frac{\sum_{y=1}^{N} \sum_{x=1}^{W} L_{x y}}{H W},
$$


where $H, W-$ a height and width of the image, and $L_{x y}$ - brightness of the element of the current image with coordinates $x$ and $y$.

Such objective characteristics as root-mean-square deviation $(\sigma)$ and entropy $(\varepsilon)$ are used for quantitate quality assessment. Root-mean-square deviation is equal to notions - local contrast and accuracy to some extent. Entropy is a measure of quantity of information in the image.

Task to estimate image quality has a multicriterion nature, so an additive generalized criterion $F$ is introduced as follows:

$$
F=\sum_{i=1}^{p} \beta_{i} f_{i},
$$

where $\beta_{i}$-weight coefficients, $\sum_{i=1}^{p} \beta_{i}=1-$ a condition of normalization $F, f_{i}$ - partial normalized criteria, $p$-a number of partial criteria.

Normalized partial indices of the contrast and numbers of informative levels are determined as follows:

$$
\begin{gathered}
K_{n}=\frac{\left(L_{\max }-L_{\min }\right)}{255}, \\
N_{n}=\frac{N}{N_{\max }},
\end{gathered}
$$

where $L_{\max }=\max \left(L_{x y}\right), \quad L_{\min }=\min \left(L_{x y}\right)-$ maximum and minimum values of brightness of image elements, $N$ - a number of informative levels being different from null, $N_{\max }=256$ - a maximum number of informative levels in digital images for visualization.

Shannon entropy estimation can be calculated for any half-tone (including television and thermal) image. In this case estimation of distribution of possibilities of gray shades in the half-tone image is calculated [3]. Calculation of the entropy is performed based on the image histogram which distribution of frequencies is described by a simple expression:

$$
p_{i}=\frac{N_{i}}{H W}
$$

where $N_{i}$ - a number of elements having $i$ level.

Calculation of the image entropy is performed according to formula:

$$
\varepsilon=-\sum_{i} p_{i} \log _{2}\left(p_{i}\right) .
$$

For normalization entropy values can be divided into a coefficient being an entropy maximum for such number of levels. For the half-tone image with 256 brightness gradations it is equal to 8 . So, the image entropy value can vary from 0 to 1 .

Image dispersion is calculated as following:

$$
\sigma^{2}=\sum_{i} p_{i}(i-\bar{i})^{2}
$$

where $\bar{i}=\sum_{i} i p_{i}, i-$ a level of quantization.

For the half-tone image estimation of dispersion of distribution of possibilities of gray shades is calculated. Experimentally it was determined on a series of different images that root-mean-square deviation varies within the range from $\approx 0$ to $\approx 100$, then the mean value is 50 , consequently $\sigma_{n}$ :

$$
\sigma_{n}=\left\{\begin{array}{l}
\frac{\sigma}{50}, \quad \sigma \leq 50, \\
\frac{(100-\sigma)}{50}, \quad 50<\sigma \leq 100, \\
0, \quad \sigma>100 .
\end{array}\right.
$$

For average brightness, values belonging to middle of the range are preferable, and on boundaries of the brightness range its

$$
\text { value is minimum: } \bar{L}_{n}=\left\{\begin{array}{l}
\frac{\bar{L}}{128}, \bar{L} \leq 107, \\
\frac{(255-\bar{L})}{128}, \quad \bar{L}>147, \\
1, \quad \bar{L} \in\{108 \div 147\} .
\end{array}\right.
$$

Entropy achieves its maximum under the uniform distribution law. Image entropy having the range from 0 to 255 brightness gradations cannot exceed 8. Normalized value of the entropy has the form: $\varepsilon_{n}=\frac{\varepsilon}{8}$. 
Main complication of particular index application is selection of weight coefficients taking into consideration influence of corresponding particular indices on the generalized criterion as a whole. Fishburne criterion is used for selection of initial values of these coefficients $[3,4]$ :

$$
\beta_{i}=\frac{2(p-i+1)}{p(p+1)}
$$

For this purpose partial criteria are divided into groups by priorities: $\left(L_{n}, \sigma_{n}\right) ;\left(K_{n}, N_{n}\right)$ and $\left(\varepsilon_{n}\right)$. Root-mean-square deviation contribution weightiness to the value of the quality function integral index is described by this index meaning: it determines accuracy and to some extent perceives intense noise. Then indices are arranged by descending of influence inside separated priority groups.

Taking into account above-mentioned facts, integral performance index (IPI) of the image brightness component has the form:

$$
\mathrm{IPI}=0,33 \bar{L}_{n}+0,27 \sigma_{n}+0,20 K_{n}+0,13 N_{n}+0,07 \varepsilon_{n} \text {. }
$$

Correction of coefficients under partial indices is performed by the method of expert evaluations, besides their amount should be equal to one.

Noise power for the whole image is calculated in the block of noise evaluation. For this purpose, image is divided into windows of size $3 \times 3$ and value of the neighbor pixel brightness which is located diagonally to the central one is subtracted from the central pixel brightness. Result of subtraction is raised to the square and summed up by all pixels of the image.

Described algorithm allows automatically selecting a method of contrasting and also a mode of interference compensation. However, usage of this algorithm on a video sequence leads to appearance of areas where a sharp jump in brightness occurs (other algorithm of contrasting is chosen), such event negatively influences on perception of video information by an operator. The method described below is suggested to be used for solution of this issue.

\section{Interpolation method of proportional application of two boundary algorithms}

Let's suppose that $k$ is a number of the video frame where replacement of the algorithm occurs, then $k+t=k^{\prime}$ is a number of the following frame where analysis and selection of the enhancement algorithm are performed, then $k_{\mathrm{T}}$ is a current video frame, besides $k \leq k_{\mathrm{T}} \leq k^{\prime}$. Let's designate $A_{k}$ as an enhancement algorithm on the $k$-frame and $A_{k^{\prime}}$ as an enhancement algorithm after $t$ frames after $k$-frame. Interpolation method is the following: for each $k_{\mathrm{T}}$ frame, proportion of two algorithms $A_{k}$ and $A_{k^{\prime}}$ is calculated, so, the closer $k_{\mathrm{T}}$ is to $k^{\prime}$, the higher coefficient the result of algorithm $A_{k^{\prime}}$ is used with, and consequently, the lower coefficient the result of algorithm $A_{k}$ is used with. Reversed situation can occur similarly when $k_{\mathrm{T}}$ is closer to boundary $k$. Hence, formula for calculation of the resulting image pixel brightness depending on value $k_{\mathrm{T}}$ has the following form:

$$
I_{x, y}=I_{x, y}^{A_{k}\left(k_{\mathrm{T}}\right)} *\left(1-\frac{k_{\mathrm{T}}-k}{t}\right)+I_{x, y}^{A_{k^{\prime}}\left(k_{\mathrm{T}}\right)} *\left(\frac{k_{\mathrm{T}}-k}{t}\right)
$$

where $\mathrm{x}, \mathrm{y}$ - pixel coordinates, $I_{x, y}^{A_{k}\left(k_{m}\right)}$ - a buffer with a result of the first algorithm for the current frame, $I_{x, y}^{A_{k^{\prime}}\left(k_{m}\right)}-$ a buffer with a result of the second algorithm for the same frame, $A_{k}\left(k_{\mathrm{T}}\right), A_{k^{\prime}}\left(k_{\mathrm{T}}\right)$ - results of operation of algorithms selected on $k$ and $k^{\prime}$ frames correspondingly, $t$ - a number of frames between moments when automatic choice of the algorithm happens.

Such formula allows gradually changing applied algorithms without sharp bursts on the resulting image but requires processing of the image by two algorithms that decreases resulting performance. The algorithm based on this method begins operation with obtaining of results of the automatic selection for $k$ and $k^{\prime}$ frames. Then each frame is processed by two algorithms $A_{k}$ and $A_{k}$, processing results are stored in two buffers of images. Each pixel of the resulting image is formed according to formula 4 . After achievement of the interval boundary $\left(k_{\mathrm{T}}=k^{\prime}\right), A_{k}=A_{k^{\prime}}$, and value $A_{k^{\prime}}$ is obtained from automatic selection of the following enhancement algorithm.

\section{Results of the algorithm for automatic selection of the enhancement method}

Fig.1 shows four frames from a video fragment and current variant of enhancement provided by the algorithm of automatic selection with interval $t=50$ frames. In this case result of the automatic selection for the $k+t$ frame is different from other frames of this sequence. Such choice leads to the fact that if brightness sharply changes on one frame of the video fragment (e.g. light flash has occurred) then enhancement of following $n$ frames is performed with the ineffectively selected algorithm. For solution of this issue stack is used. The stack contains four best elements of the set $\mathrm{M}$ which were obtained as a result of preliminary operation of the algorithm. When stack is filled completely, selection of the enhancement algorithm is performed by calculating frequencies of repetitions $A_{i}$ and $B_{j}$ in this stack. For sequence in Fig.1 the following repetition frequencies are obtained:

$$
\mathrm{F}\left(\mathrm{A}_{2}\right)=1 ; \mathrm{F}\left(\mathrm{A}_{3}\right)=3 ; \mathrm{F}\left(\mathrm{B}_{0}\right)=4
$$

So, the best element is $\left(\mathrm{A}_{3}, \mathrm{~B}_{0}\right) \in \mathrm{M}$. 


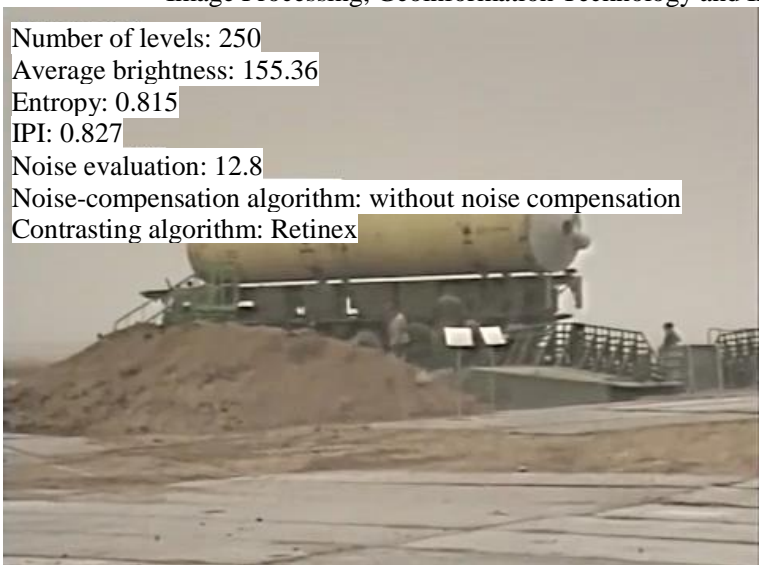

$k$ frame $\left(\mathrm{A}_{3}, \mathrm{~B}_{0}\right)$

Number of levels: 230

Average brightness: 135.77

Entropy: 0.799

IPI: 0.852

Noise evaluation: 8.37

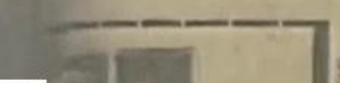

Noise-compensation algorithm: without noise compensation

Contrasting algorithm: Retinex

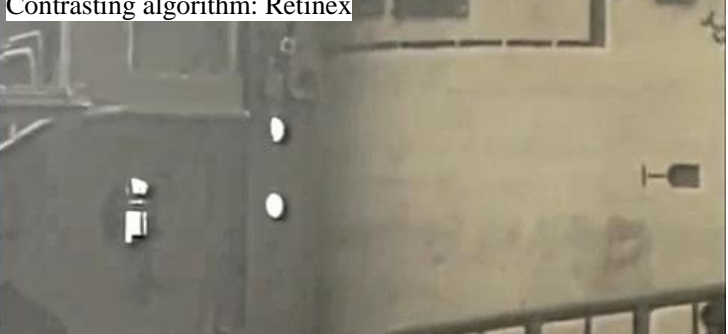

$(k+2 * n)$ frame $\left(\mathrm{A}_{3}, \mathrm{~B}_{0}\right)$
Number of levels: 152

Average brightness: 129.02

Entropy: 0.84

IPI: 0.803

Noise evaluation: 10

Noise-compensation algorithm: without noise compensation

Contrasting algorithm: histogram equalization

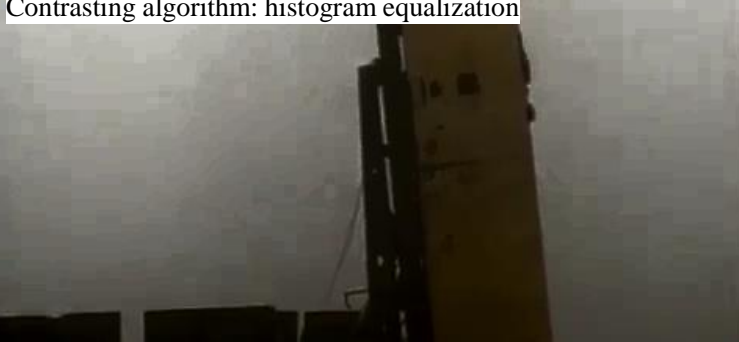

$(k+t)$ frame $\left(\mathrm{A}_{2}, \mathrm{~B}_{0}\right)$

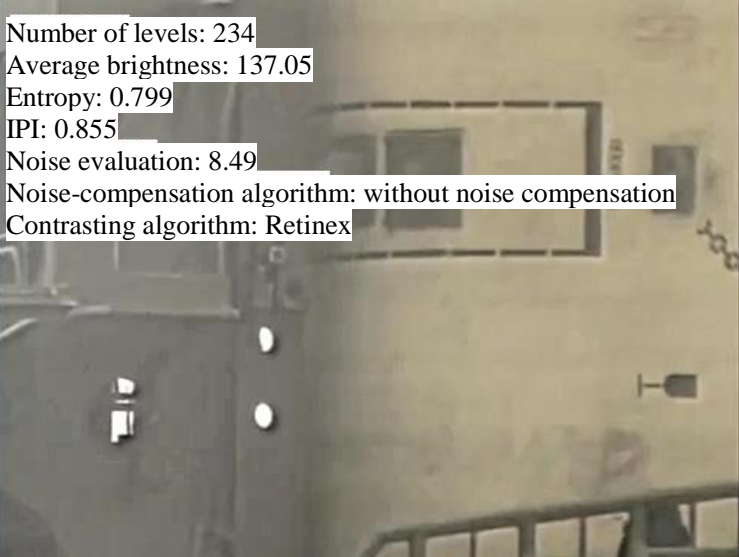

$(k+3 * n)$ frame $\left(\mathrm{A}_{3}, \mathrm{~B}_{0}\right)$

Fig. 1. Results of four frame evaluation

Let's consider the same four frames but now with application of the stack. Result is shown in Fig.2.

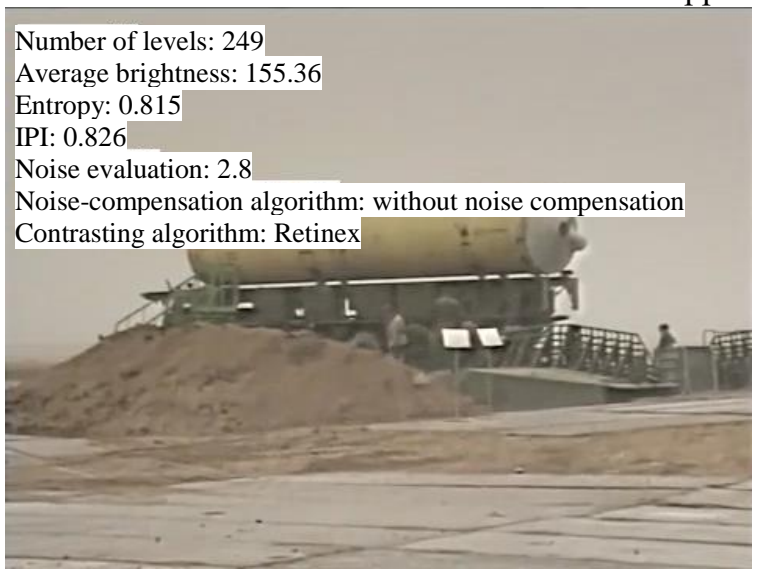

$k$ frame $\left(\mathrm{A}_{3}, \mathrm{~B}_{0}\right)$

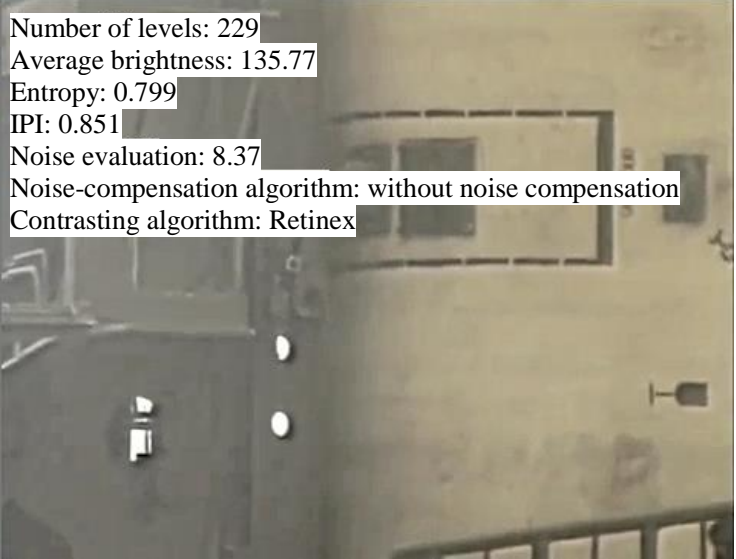

$(k+2 * n)$ frame $\left(\mathrm{A}_{3}, \mathrm{~B}_{0}\right)$
Number of levels: 207

Average brightness: 149.63

Entropy: 0.725

IPI: 0.767

Noise evaluation: 7.25

Noise-compensation algorithm: without noise compensation

Contrasting algorithm: Retinex

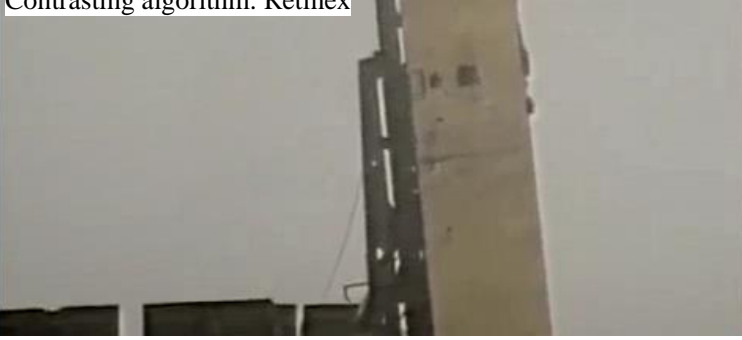

$(k+n)$ frame $\left(\mathrm{A}_{3}, \mathrm{~B}_{0}\right)$

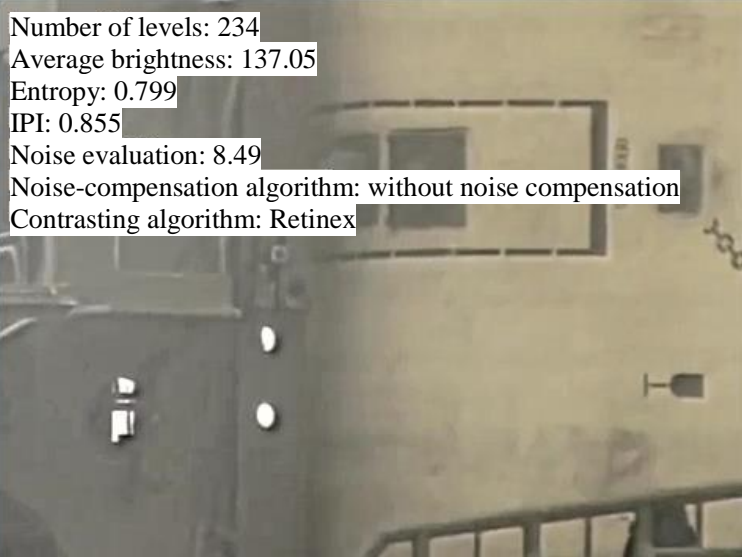

$(k+3 * n)$ frame $\left(\mathrm{A}_{3}, \mathrm{~B}_{0}\right)$

Fig.2. Result of the algorithm operation for automatic selecting using a stack. 
We can see that the frame with number $(k+t)$ is now processed by the same sequence of algorithms as other frames. It allows avoiding undesirable darkening.

Fig. 3 shows a diagram of dependence of IPI on a number of the frame. Numbers of frames are marked in horizontal direction and values of quality indices are marked in vertical direction, A0-A3 - contrasting algorithms (A0 -without contrasting, A1 linear stretch of the histogram [5], A2 - histogram equalization [6], A3 - Multi Scale Retinex with Color Restoration [7]), Auto - IPI values under automatic selection of contrasting algorithms.

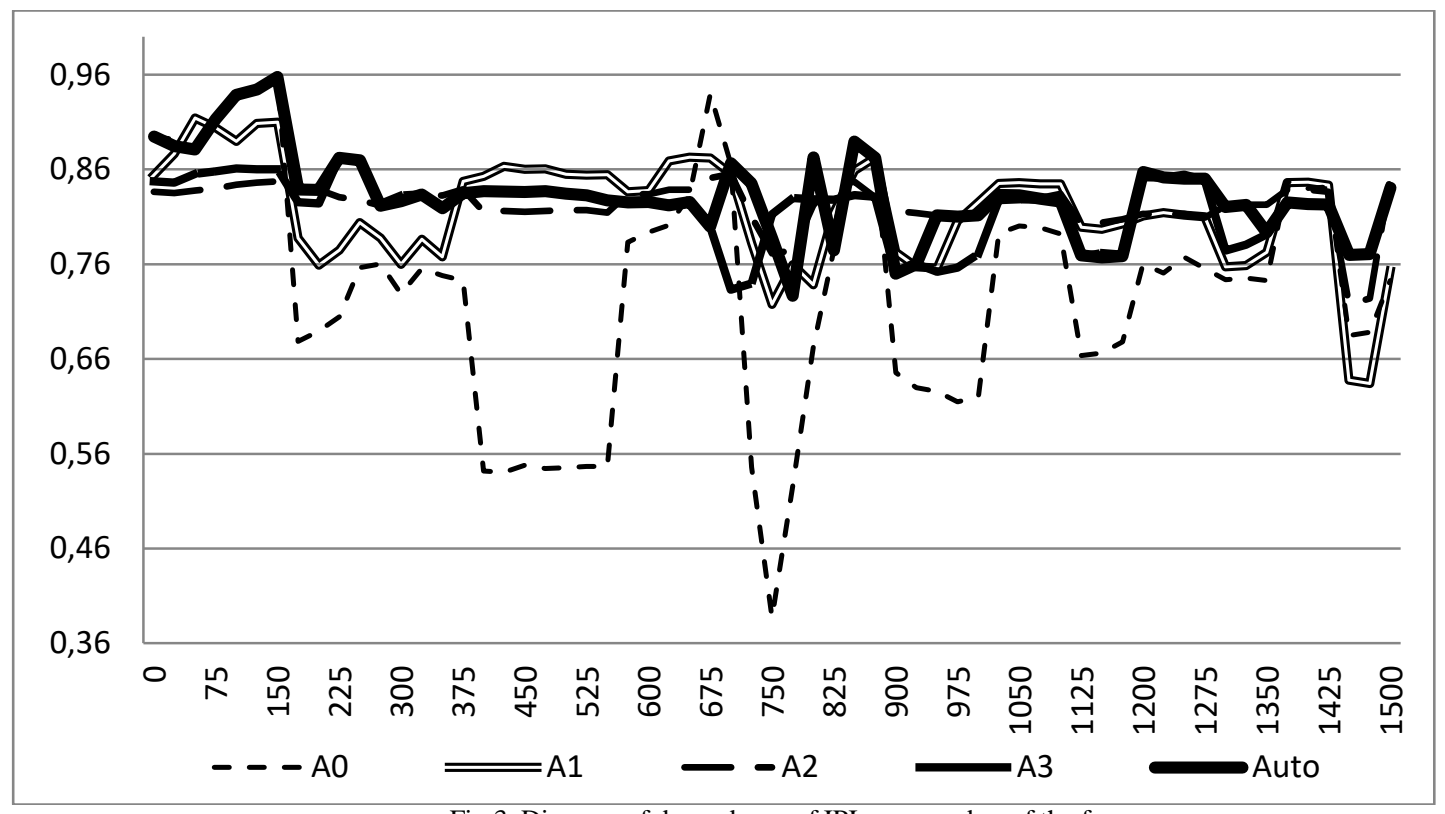

Fig.3. Diagram of dependence of IPI on a number of the frame.

On the diagram we can see areas which value of IPI has not the best value for, it is described by delay occurred because the stack is used and evaluation is performed after every $t=50$ frames.

\section{Conclusion}

Application of the suggested algorithm for automatic selection of the enhancement method allows automatically finding the best combination of methods for image enhancement. The algorithm provides a possibility to change both a number of used methods of enhancement and also methods for image evaluation not causing significant changes in the algorithm structure. It allows implementing new enhancement algorithms and also new methods of evaluation. Application of the stack for accumulation of processing results and interpolation method of proportional application of two boundary algorithms allows avoiding errors in choice of the best algorithms.

\section{References}

[1] Elesina SI. Imposition of images in correlation-extreme navigation systems. Edited by Kostyashkina LN, Nikiforov MB. Moscow: Radio Engineering, 2015; 208 p.

[2] Wang Z, Bovik AC, Sheikh HR, Simoncelli EP. Image quality assessment: From error visibility to structural similarity. IEEE Trans. Image Process. 2004; 13(4): 600-612.

[3] Gurov VS. Image processing in aviation vision systems: monograph. Edited by Kostyashkina LN, Nikiforov MB. Moscow: FIZMATLIT, 2016; 240p.

[4] Fishburne PS. Theory of utility for decision-making. Moscow: Nauka, 1978; 352 p.

[5] Gonzalez RC, Woods RE. Digital Image Processing. Prentice Hall, 2 edition, 2002.

[6] Singh RP, Dixit M. Histogram Equalization: A Strong Technique for Image Enhancement. International Journal of Signal Processing, Image Processing and Pattern Recognition 2015; 8(8): 345-352.

[7] Petro AB, Sbert CS, Morel JM. Multiscale Retinex. Image Processing On Line 2014; 4: 71-88. 